\title{
Fine-needle aspiration of a retropharyngeal lymph node guided by endoscopic ultrasonography
}
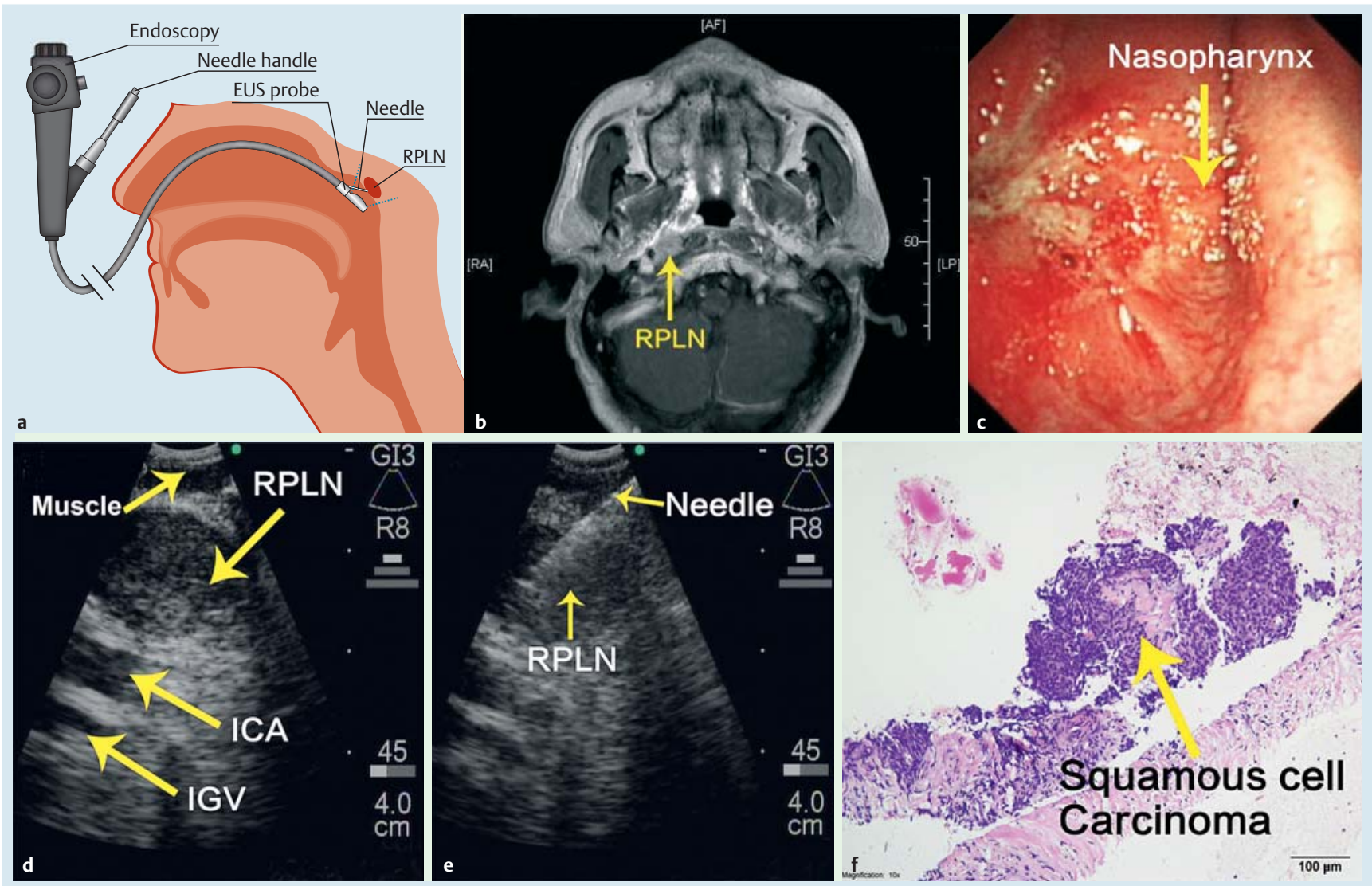

Fig. 1 a Schematic diagram of endoscopic ultrasound-guided fine-needle aspiration (EUS-FNA) of a retropharyngeal lymph node (RPLN) in a 50-year-old patient with suspected recurrence of nasopharyngeal carcinoma. The EUS needle is introduced into the nasopharynx, and sampling through the nasopharynx under EUS guidance provides the shortest puncture path and avoids several crucial tissues and organs. b T1-weighted contrast-enhanced magnetic resonance imaging shows an enlarged retropharyngeal lymph node (RPLN) in a patient with suspected recurrence of nasopharyngeal carcinoma. $\mathbf{c}$ Post-radiotherapy changes, with nasopharyngeal mucosal roughness and local scarring, are seen on nasopharyngoscopy. $\mathbf{d}$ An enlarged retropharyngeal lymph node (RPLN), which is roughly round and hypoechoic, is visualized by EUS. The RPLN is adjacent to the carotid sheath, which contains the internal carotid artery (ICA) and internal jugular vein (IGV). e Procedure of EUS-FNA in a retropharyngeal lymph node (RPLN). The needle penetrates the RPLN while visualized and monitored by real-time EUS, and biopsy tissue is obtained by EUS-FNA. $\mathbf{f}$ A squamous cell carcinoma nest is visualized in a biopsy specimen from the retropharyngeal lymph node. Consequently, recurrence of nasopharyngeal carcinoma is confirmed.

Recently, we developed a novel, minimally invasive technique - endoscopic ultrasonography-guided fine-needle aspiration (EUS-FNA) via the nasopharynx - to obtain tissue samples from a retropharyngeal lymph node (RPLN) in a patient with suspected recurrent nasopharyngeal carcinoma [1]. A schematic diagram of EUSFNA of an RPLN is shown in 1 Fig. 1 a.

In April 2015, a 50-year-old man who had received chemoradiotherapy for nasopharyngeal carcinoma 2 years earlier was admitted to the Sun Yat-sen University Cancer Center. Magnetic resonance imaging displayed an enlarged RPLN on the right side, $2.0 \times 1.6 \mathrm{~cm}$ in size and with central enhancement on T1-weighted contrast-enhanced imaging ( $\mathbf{F i g . 1 \mathbf { b } )}$ [2]. Nasopharyngoscopy revealed nasopharyngeal mucosal roughness and local scarring on the right ( $\nabla$ Fig. 1 c). Multiple mucosal biopsy specimens were negative for cancer cells. After a multidisciplinary consultation, the patient was advised to undergo EUS-FNA.

An EUS instrument (BF-UC 260F-OL8; Olympus, Tokyo, Japan) was introduced via the right nostril and nasopharynx to scan the retropharyngeal space. Ultrasonography revealed an enlarged RPLN with an axial diameter of $2.0 \mathrm{~cm}$ on the right side of the retropharyngeal space. The RPLN was close to the carotid sheath, which contained the internal carotid artery and the internal jugular vein [3]. The mass was roughly round and homogeneously hypoechoic ( $\bullet$ Fig. 1 d).

Under real-time EUS guidance, a dedicated 22-gauge aspiration needle (NA-201SX4022; Olympus) was used to puncture the enlarged RPLN; the needle was then withdrawn under $10 \mathrm{~mL}$ of suction pressure ( $\bullet$ Fig. 1e). The obtained tissue samples were sent for pathological and cytological examination. This EUS-FNA procedure 


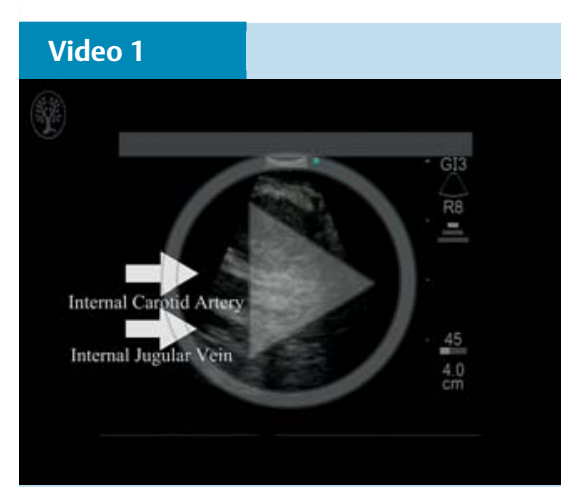

Endoscopic ultrasound-guided fine-needle aspiration of a retropharyngeal lymph node.

was repeated three times and lasted for a total of approximately 20 minutes [4]. The procedure of EUS-FNA of an RPLN is shown in 0 Video 1.

The EUS-FNA procedure was conducted smoothly without any severe complication, such as bleeding, subcutaneous emphysema, choking, dyspnea, extremity paralysis, or hemiplegia. The pathological result confirmed the presence of squamous cell metastases in the RPLN (o Fig. 1f) [5].

Endoscopy_UCTN_Code_TTT_1AS_2AB

Competing interests: None

\section{Jian-Jun Li', Long-Jun He', Guang-Yu Luo', Li-Zhi Liu'², Xin-Xin Huang', Ke Pan ${ }^{3}$, Guo-Liang $\mathrm{Xu}^{1}$}

${ }^{1}$ Department of Endoscopy, Sun Yat-sen University Cancer Center, State Key Laboratory of Oncology in South China, Collaborative Innovation Center for Cancer Medicine, Guangzhou, P. R. China

2 Department of Imaging and Intervention Radiology, Sun Yat-sen University Cancer Center, State Key Laboratory of Oncology in South China, Collaborative Innovation Center for Cancer Medicine, Guangzhou, P. R. China

${ }^{3}$ Department of Experiment, Sun Yat-sen University Cancer Center, State Key Laboratory of Oncology in South China, Collaborative Innovation Center for Cancer Medicine, Guangzhou, P. R. China

\section{References}

1 Ma J, Liu L, Tang $L$ et al. Retropharyngeal lymph node metastasis in nasopharyngeal carcinoma: prognostic value and staging categories. Clin Cancer Res 2007; 13: $1445-1452$

2 Zhang GY, Liu LZ, Wei WH et al. Radiologic criteria of retropharyngeal lymph node metastasis in nasopharyngeal carcinoma treated with radiation therapy. Radiology 2010; 255: 605-612
3 Chan JY, Chow VL, Wong ST et al. Surgical salvage for recurrent retropharyngeal lymph node metastasis in nasopharyngeal carcinoma. Head Neck 2013; 35: 1726-1731

4 Grosu HB, Morice RC, Sarkiss M et al. Safety of flexible bronchoscopy, rigid bronchoscopy, and endobronchial ultrasound-guided transbronchial needle aspiration in patients with malignant space-occupying brain lesions. Chest 2015; 147: $1621-1628$

5 Su Y, Zhao C, Li WJ et al. CT-guided needle biopsy through mandibular area for the diagnosis of nasopharyngeal carcinoma in the parapharyngeal space. Chin J Cancer 2010; 29: $768-773$

\section{Bibliography}

Dol http://dx.doi.org/

10.1055/s-0034-1392652

Endoscopy 2015; 47: E449-E450

(c) Georg Thieme Verlag KG

Stuttgart · New York

ISSN 0013-726X

\section{Corresponding author}

\section{Jian-Jun Li, MD}

Department of Endoscopy

Sun Yat-sen University Cancer Center

Dongfeng Road East

Guangzhou 510060

P. R. China

Fax: +86-20-87343224

lijj@sysucc.org.cn 